\title{
Articles of Significant Interest in This Issue
}

\author{
Components Responsible for Intracellular Activity of Pasteurella multocida \\ Toxin Are Delivered to the Cytosol as an Intact Multidomain Cargo
}

Pasteurella multocida toxin (PMT) is an A-B toxin that mediates its cytotoxicity through deamidation of cytosolic heterotrimeric G-protein targets. Clemons et al. (e00248-18) demonstrate that the $\mathrm{N}$ terminus of PMT (PMT-N) mediates cytosolic delivery of native C-terminal cargo as an intact unit comprised of subdomains C1-C2-C3, including the membrane localization subdomain $\mathrm{C} 1$ and the catalytic subdomain C3. Although PMT-N can deliver nonnative green fluorescent protein cargo alone and can deliver C1-C2 without C3, cytosolic delivery of C3 requires that C3 is packaged with C1-C2 as a unit.

\begin{abstract}
A Capsule-Targeting Glycoside Hydrolase Offers Protection from Pneumococcal Infection

Invasive pneumococcal diseases caused by Streptococcus pneumoniae have been a major threat to human health, with alarming mortality rates. Despite a global vaccination program and the use of antibiotics, S. pneumoniae remains among the deadliest infectious agents worldwide. Middleton et al. (e00316-18) demonstrate the protective role of a glycoside hydrolase, Pn3Pase, targeting the capsule of highly pathogenic serotype 3 of S. pneumoniae. In vivo degradation of capsule by Pn3Pase dramatically reduces colonization and protects mice from sepsis caused by type 3 S. pneumoniae. This work validates an alternative targeted therapeutic approach to the shortcomings of the incumbent vaccine and antibiotic solutions to invasive pneumococcal disease.
\end{abstract}

Candida albicans Cannot Acquire Sufficient Host Ethanolamine To Support Virulence

Candida albicans is a common human fungal pathogen causing mucosal and systemic infections. The cho $1 \Delta \Delta$ and psd $1 \Delta \Delta$ psd $2 \Delta \Delta$ mutants cannot synthesize phosphatidylethanolamine from phosphatidylserine and are avirulent in mice, even though these mutants can produce phosphatidylethanolamine from imported ethanolamine. As ethanolamine is present in host animals, it is possible that $C$. albicans cannot acquire ethanolamine from the host in sufficient amounts. To test this hypothesis, Davis et al. (e00815-17) engineered cho $1 \Delta \Delta$ and psd $1 \Delta \Delta$ psd $2 \Delta \Delta$ mutants to express the Arabidopsis thaliana serine decarboxylase (AtSDC) plant enzyme, which synthesizes ethanolamine cytoplasmically, bypassing the need to import host ethanolamine. Phosphatidylethanolamine synthesis and full virulence were restored in AtSDC-expressing mutants. Thus, C. albicans cannot acquire sufficient host ethanolamine to support pathogenicity.

\begin{abstract}
Human Monoclonal Antibodies for Immunoprophylaxis against Enterotoxigenic Escherichia coli Infection

Infection with enterotoxigenic Escherichia coli (ETEC) is a global health problem and a major cause of diarrheal illness in infants in the developing world and travelers to countries where the disease is endemic, including military personnel. There is currently no vaccine licensed to prevent ETEC. Diarrhea follows adhesion of ETEC to intestinal cells through colonization factor antigen (CFA). Giuntini et al. (e00355-18) characterized human monoclonal antibodies (HuMAbs) against one of the most common CFAs (CfaE). Oral administration of anti-CfaE antibodies, either lgG or secretory $\lg \mathrm{A}$, inhibited intestinal colonization in mice challenged with ETEC. This work supports the potential for the development of HuMAbs as an oral immunoprophylactic to prevent ETEC-related diarrhea.
\end{abstract}

